\title{
BI-MAGIC AND OTHER GENERALIZATIONS OF SUPER EDGE-MAGIC LABELINGS
}

\author{
S. C. LÓPEZ ${ }^{\bowtie}$, F. A. MUNTANER-BATLE and M. RIUS-FONT
}

(Received 27 November 2010)

Dedicated to the memory of Professor Gary Bloom

\begin{abstract}
In this paper, we use the product $\otimes_{h}$ in order to study super edge-magic labelings, bi-magic labelings and optimal $k$-equitable labelings. We establish, with the help of the product $\otimes_{h}$, new relations between super edge-magic labelings and optimal $k$-equitable labelings and between super edge-magic labelings and edge bi-magic labelings. We also introduce new families of graphs that are inspired by the family of generalized Petersen graphs. The concepts of super bi-magic and $r$-magic labelings are also introduced and discussed, and open problems are proposed for future research.
\end{abstract}

2010 Mathematics subject classification: primary 05C78.

Keywords and phrases: super edge-magic, bi-magic, $k$-equitable.

\section{Introduction}

For most of the graph-theory terminology and notation utilized in this paper we follow either [5] or [14], unless otherwise specified. In particular we may allow graphs to have loops; however no multiple edges will be allowed unless we are in Section 4. Let $G=(V, E)$ be a graph. We say that a graph $G$ is a $(p, q)$-graph if $|V|=p$ and $|E|=q$. Kotzig and Rosa introduced in [10] the concept of edge-magic labeling. A bijective function $f: V \cup E \longrightarrow\{i\}_{i=1}^{p+q}$ is an edge-magic labeling of $G$ if there exists an integer $k$ such that the sum $f(x)+f(x y)+f(y)=k$ for all $x y \in E$. In 1998, Enomoto et al. [6] defined the concepts of super edge-magic graphs and super edgemagic labelings. A super edge-magic labeling is an edge-magic labeling with the extra condition that $f(V)=\{i\}_{i=1}^{p}$. It is worthwhile mentioning that an equivalent labeling had already appeared in the literature in 1991 under the name of strongly indexable labeling [1]. A graph that admits a (super) edge-magic labeling is called a (super) edge-magic graph.

The research conducted in this document by first and third author has been supported by the Spanish Research Council under project MTM2008-06620-C03-01 and by the Catalan Research Council under grant 2009SGR1387.

(C) 2011 Australian Mathematical Publishing Association Inc. 0004-9727/2011 \$16.00 
In 2000, Figueroa et al. [7] provided a very useful characterization of super edgemagic graphs that we state in the next lemma.

LEMMA 1.1. A ( $p, q)$-graph $G$ is super edge-magic if and only if there is a bijective function $\bar{f}: V \longrightarrow\{i\}_{i=1}^{p}$ such that the set $S_{E}=\{\bar{f}(u)+\bar{f}(v): u v \in E\}$ is a set of $q$ consecutive integers.

In [8], Figueroa et al. introduced the concept of super edge-magic digraph as follows: a digraph $D=(V, E)$ is super edge-magic if its underlying graph is super edge-magic. In general, we say that a digraph $D$ admits a labeling $f$ if its underlying graph admits the labeling $f$. In this paper we will use super edge-magic digraphs in order to achieve our goals. In [4] Bloom and Ruiz introduced a generalization of graceful labelings (see [9] for a formal definition of graceful labeling), which they called $k$-equitable labelings. Let $G=(V, E)$ be a $(p, q)$-graph and let $g: V \longrightarrow \mathbb{Z}$ be an injective function with the property that the new function $h: E \longrightarrow \mathbb{N}$ defined by the rule $h(u v)=|g(u)-g(v)|$ for all $u v \in E$ assigns the same integer to exactly $k$ edges. Then $g$ is said to be a $k$-equitable labeling and $G$ a $k$-equitable graph. In [4] the authors called a $k$-equitable labeling, optimal, when $g$ assigns all the elements of the set $\{i\}_{i=1}^{p}$ to the elements of $V$. Both Bloom and Wojciechowski $[15,16]$, and independently Barrientos [2], proved that $C_{n}$ is optimal $k$-equitable if and only if $k$ is a proper divisor of $n(k \neq n)$.

From now on, we will use the notation und $(D)$ in order to denote the underlying graph of a digraph $D$. At this point let $D=(V, E)$ with $V \subset \mathbb{N}$ be any digraph. We define the adjacency matrix of $D$, denoted by $A(D)$, to be the matrix such that the rows and columns are named after the vertices of $D$ in increasing order, and an entry $(i, j)$ of the matrix is 1 if and only if $(i, j) \in E$. Otherwise, the entry $(i, j)$ is 0 .

In [8], Figueroa et al. defined the following product: let $D=(V, E)$ be a digraph with adjacency matrix $A(D)=\left(a_{i, j}\right)$ and let $\Gamma=\left\{F_{i}\right\}_{i=1}^{m}$ be a family of $m$ digraphs with the same set of vertices $V^{\prime}$. Assume that $h: E \longrightarrow \Gamma$ is any function that assigns elements of $\Gamma$ to the arcs of $D$. Then the digraph $D \otimes_{h} \Gamma$ is defined by the following:

(1) $V\left(D \otimes_{h} \Gamma\right)=V \times V^{\prime}$;

(2) $\quad\left(\left(a_{1}, b_{1}\right),\left(a_{2}, b_{2}\right)\right) \in E\left(D \otimes_{h} \Gamma\right) \Longleftrightarrow\left[\left(a_{1}, a_{2}\right) \in E(D) \wedge\left(b_{1}, b_{2}\right) \in\right.$ $\left.E\left(h\left(a_{1}, a_{2}\right)\right)\right]$.

An alternative way of defining the same product is through adjacency matrices, since we can obtain the adjacency matrix of $D \otimes_{h} \Gamma$ as follows.

(1) If $a_{i, j}=0$ then $a_{i, j}$ is multiplied by the $p^{\prime} \times p^{\prime} 0$-square matrix.

(2) If $a_{i, j}=1$ then $a_{i, j}$ is multiplied by $A(h(i, j))$ where $A(h(i, j))$ is the adjacency matrix of the digraph $h(i, j)$.

Note that when $h$ is constant, $D \otimes_{h} \Gamma$ is the Kronecker product. From now on, let $S_{n}$ denote the set of all super edge-magic 1-regular labeled digraphs of order $n$ where each vertex takes the name of the label that has been assigned to it. We also denote by $\Sigma_{n}$ the set of all 1-regular digraphs of order $n$. 
The following results were introduced in [8].

THEOREM 1.2. Let $D$ be a (super) edge-magic digraph and let $h: E(D) \longrightarrow S_{n}$ be any function. Then und $\left(D \otimes_{h} S_{n}\right)$ is (super) edge-magic.

THEOREM 1.3. Let $\overrightarrow{C_{m}}$ be a strong orientation of $C_{m}$ and let $h: E\left(\overrightarrow{C_{m}}\right) \longrightarrow S_{n}$ be any constant function. Then und $\left(\overrightarrow{C_{m}} \otimes_{h} S_{n}\right)=\operatorname{gcd}(m, n) C_{\mathrm{lcm}[m, n]}$.

THEOREM 1.4. Let $F$ be an acyclic graph. Consider any function $h: E(\vec{F}) \longrightarrow \Sigma_{n}$. Then und $\left(\vec{F} \otimes_{h} \Sigma_{n}\right)=n F$.

Using this product, in the original paper, Figueroa et al. were able to find exponential lower bounds for the number of nonisomorphic labelings of different types, and different families of graphs.

\section{Generalizations of generalized Petersen graphs and the $\otimes_{h}$-product}

The generalized Petersen graph $P(n ; k), n \geq 3$ and $1 \leq k \leq\lceil(n-1) / 2\rceil$, consists of an outer $n$-cycle $x_{0} x_{1} \cdots x_{n-1} x_{0}$, a set of $n$-spokes $x_{i} y_{i}, 0 \leq i \leq n-1$, and $n$ inner edges of the form $y_{i} y_{i+{ }_{n} k}$, where ${ }_{{ }_{n}}$ denotes the sum of two elements in the group $\mathbb{Z}_{n}$. In this section we propose two possible generalizations of this family, one replacing the $k$ step of the inner edges by a permutation and another one, increasing the number of levels. We denote by $\mathfrak{S}_{n}$ the set of permutations of $\{0,1, \ldots, n-1\}$.

Let $n \geq 3$ and let $\pi \in \mathfrak{S}_{n}$. The first generalization of a generalized Petersen graph considered in this paper $\operatorname{GGP}(n ; \pi)$, consists of an outer $n$-cycle $x_{0} x_{1} \cdots x_{n-1} x_{0}$, a set of $n$-spokes $x_{i} y_{i}, 0 \leq i \leq n-1$ and $n$ inner edges defined by $y_{i} y_{\pi(i)}, i=$ $0, \ldots, n-1$. Notice that, if we consider the permutation $\pi$ defined by $\pi(i)=i+{ }_{n} k$ then $G G P(n ; \pi)=P(n ; k)$.

Let $m \geq 2, n \geq 3$ and $\pi_{2}, \ldots, \pi_{m} \in \mathfrak{S}_{n}$. The second generalization of $a$ generalized Petersen graph considered in this paper $\operatorname{GGP}\left(n ; \pi_{2}, \ldots, \pi_{m}\right)$ is a graph with vertex set $\bigcup_{j=1}^{m}\left\{x_{i}^{j}: i=0, \ldots, n-1\right\}$, an outer $n$-cycle $x_{0}^{1} x_{1}^{1} \cdots x_{n-1}^{1} x_{0}^{1}$, and inner edges $x_{i}^{j-1} x_{i}^{j}$ and $x_{i}^{j} x_{\pi_{j}(i)}^{j}$, for $j=2, \ldots, m$, and $i=0, \ldots, n-1$. Notice that, $G G P\left(n ; \pi_{2}, \ldots, \pi_{m}\right)=P_{m} \times C_{n}$, when $\pi_{j}(i)=i+{ }_{n} 1$ for every $j=2, \ldots, m$.

The graphs $\operatorname{GGP}(9 ; \pi)$ and $\operatorname{GGP}\left(5 ; \pi_{2}, \pi_{3}\right)$ are shown in Figure 1 , where $\pi \in \mathfrak{S}_{9}, \pi_{2}, \pi_{3} \in \mathfrak{S}_{5}$ and $\pi=(0,1,8,3,4,2,6,7,5), \pi_{2}=(0,2,4,1,3)$ and $\pi_{3}(i)=i+{ }_{5} 1$.

Let $\overrightarrow{L P_{m}}$ be the digraph obtained from a path of $m$-vertices, in such a way that we can travel from one leaf to the other following the directions of the arrows, with a loop attached at each vertex.

PROPOSITION 2.1. Let $\overrightarrow{C_{n}}$ be a strong connected digraph obtained from a cycle of order $n$ where $n$ is odd. Then

$$
\operatorname{und}\left(\overrightarrow{L P_{m}} \otimes \overrightarrow{C_{n}}\right)=P_{m} \times C_{n}
$$





FIGURE 1. The graphs $G G P(9 ; \pi)$ and $G G P\left(5 ; \pi_{2}, \pi_{3}\right)$.

PROOF. By definition,

$$
V\left(\overrightarrow{L P_{m}} \otimes \overrightarrow{C_{n}}\right)=V\left(P_{m} \times C_{n}\right) .
$$

Let $a_{0} a_{1} \cdots a_{m-1}$ and $b_{0} b_{1} \cdots b_{n-1}$ be directed paths respectively in $\overrightarrow{L P_{m}}$ and $\overrightarrow{C_{n}}$. Then $\left(\left(a_{i}, b_{j}\right),\left(a_{i^{\prime}}, b_{j^{\prime}}\right)\right)$ is an arc in $\overrightarrow{L P_{m}} \otimes \overrightarrow{C_{n}}$ if and only if $\left(a_{i}, a_{i^{\prime}}\right) \in E\left(\overrightarrow{L P_{m}}\right)$ and $j^{\prime}=j+{ }_{n} 1$. That is, all arcs are of the form either $\left(\left(a_{i}, b_{j}\right),\left(a_{i}, b_{j+{ }_{n} 1}\right)\right)$ or $\left(\left(a_{i}, b_{j}\right),\left(a_{i+m} 1, b_{j+{ }_{n} 1}\right)\right)$.

From now on, let us denote by $\sigma_{k} \in \mathfrak{S}_{n}$ the permutation defined by $\sigma_{k}(i)=i+{ }_{n} k$. Proposition 2.2. Let $n$ be an odd integer and let $\pi \in \mathfrak{S}_{n}$. Assume that, for some $h: E\left(\overrightarrow{L P_{2}}\right) \longrightarrow S_{n}$, we obtain

$$
\operatorname{und}\left(\overrightarrow{L P_{2}} \otimes_{h} S_{n}\right)=G G P(n ; \pi)
$$

Then there exists $h^{\prime}: E\left(\overrightarrow{L P_{m}}\right) \longrightarrow S_{n}$ such that

$$
\operatorname{und}\left(\overrightarrow{L P_{m}} \otimes_{h^{\prime}} S_{n}\right)=G G P(n ; \overbrace{\sigma_{1}, \ldots, \sigma_{1}}^{(m-2)}, \pi) \text { times }
$$

PROOF. Let $a_{0} a_{1} \cdots a_{m-1}$ and $b_{0} b_{1}$ be the directed paths induced respectively in $\overrightarrow{L P_{m}}$ and $\overrightarrow{L P_{2}}$. Let $h^{\prime}: E\left(\overrightarrow{L P_{m}}\right) \longrightarrow S_{n}$ be the function defined by

$$
h^{\prime}(e)= \begin{cases}h\left(b_{1} b_{1}\right) & \text { if } e=a_{m-1} a_{m-1}, \\ h\left(b_{0} b_{1}\right) & \text { if } e=a_{m-2} a_{m-1}, \\ h\left(b_{0} b_{0}\right) & \text { otherwise }\end{cases}
$$

Then

$$
\operatorname{und}\left(\overrightarrow{L P_{m}} \otimes_{h^{\prime}} S_{n}\right)=G G P(n ; \overbrace{\sigma_{1}, \ldots, \sigma_{1}}^{(m-2)}, \pi) \text { times }
$$

This concludes the proof. 


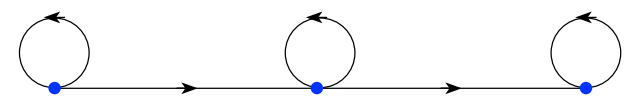

FIgURE 2. The digraph $\overrightarrow{\mathrm{LP}_{3}}$.

We can introduce a slight modification in $h^{\prime}$ in order to construct, for each $l<m$, $G G P\left(n ; \pi_{2}, \ldots, \pi_{m}\right)$, where $\pi_{i}=\sigma_{1}$ for $i \neq l$ and $\pi_{l}=\pi$.

Proposition 2.3. Let $n$ be an odd integer. Assume that, for some $h: E\left(\overrightarrow{L P_{2}}\right) \longrightarrow$ $S_{n}$, we obtain

$$
\operatorname{und}\left(\overrightarrow{L P_{2}} \otimes_{h} S_{n}\right)=G G P(n ; \pi)
$$

Then for each $l, 1<l \leq m$, there exists $h_{l}^{\prime}: E\left(\overrightarrow{L P_{m}}\right) \longrightarrow S_{n}$ such that

$$
\operatorname{und}\left(\overrightarrow{L P_{m}} \otimes_{h_{l}^{\prime}} S_{n}\right)=G G P\left(n ; \pi_{2}, \ldots, \pi_{m}\right) \text {, }
$$

where $\pi_{i}=\sigma_{1}$ for $i \neq l$ and $\pi_{l}=\pi$.

PROOF. The result follows from Proposition 2.2 when $l=m$. Hence, we only need to consider the case when $l<m$. Let $a_{0} a_{1} \cdots a_{m-1}$ and $b_{0} b_{1}$ be the directed paths induced respectively in $\overrightarrow{L P_{m}}$ and $\overrightarrow{L P_{2}}$. Assume that $\Gamma \in S_{n}$ and denote by $\stackrel{\Gamma}{\Gamma}$ the oriented digraph obtained from $\Gamma$ by reversing all its arcs. Let $h_{l}^{\prime}: E\left(\overrightarrow{L P_{m}}\right) \longrightarrow S_{n}$ be the function defined by

$$
h_{l}^{\prime}(e)= \begin{cases}h\left(b_{1} b_{1}\right) & \text { if } e=a_{l-1} a_{l-1}, \\ h\left(b_{0} b_{1}\right) & \text { if } e=a_{l-2} a_{l-1}, \\ h\left(b_{0} b_{0}\right) & \text { if } e=a_{l-2} a_{l-2}, \\ \overleftarrow{h\left(b_{0} b_{1}\right)} & \text { if } e=a_{l-1} a_{l}, \\ h\left(b_{0} b_{0}\right) & \text { otherwise. }\end{cases}
$$

Then

$$
\operatorname{und}\left(\overrightarrow{L P_{m}} \otimes_{h_{l}^{\prime}} S_{n}\right)=G G P\left(n ; \pi_{2}, \ldots, \pi_{m}\right),
$$

where $\pi_{i}=\sigma_{1}$ for $i \neq l$ and $\pi_{l}=\pi$.

Let $x_{0} x_{1} \cdots x_{m-1} x_{0}$ be the outer cycle of $P(m ; k)$ with spokes $x_{i} y_{i}, 0 \leq i \leq m-1$, and inner edges $y_{i} y_{i+m}$. We denote by $\overrightarrow{P(m ; k)}$ the oriented graph obtained from $P(m ; k)$ by orienting the edges of the outer cycle from $x_{i}$ to $x_{i+{ }_{m}}$, the inner edges from $y_{i}$ to $y_{i+{ }_{m} k}$ and the spokes from the outer cycle to the inner one.

PROPOSITION 2.4. Let $m, n$ be two positive integers such that $\operatorname{gcd}(m, n)=1$ with $n$ odd. Then

$$
\operatorname{und}\left(\overrightarrow{P(m ; k)} \otimes \overrightarrow{C_{n}}\right)=P(m n ; k+m r),
$$

where $r$ is the smallest positive integer such that $k+{ }_{n} m r=1$. 
PROOF. Let $v_{0} v_{1} \cdots v_{n-1} v_{0}$ be the cycle $\overrightarrow{C_{n}}$, where each vertex is identified with the corresponding label of a super edge-magic labeling of $\overrightarrow{C_{n}}$. Then

$$
V\left(\overrightarrow{P(m ; k)} \otimes \overrightarrow{C_{n}}\right)=\left\{\left(x_{i}, v_{j}\right),\left(y_{i}, v_{j}\right)\right\}_{i=0, \ldots, m-1}^{j=0, \ldots, n-1}
$$

and

$$
\begin{gathered}
E\left(\overrightarrow{P(m ; k)} \otimes \overrightarrow{C_{n}}\right)=\left\{\left(\left(x_{i}, v_{j}\right),\left(x_{i+{ }_{m} 1}, v_{j+{ }_{n} 1}\right)\right),\left(\left(y_{i}, v_{j}\right),\left(y_{i+{ }_{m} k}, v_{j+{ }_{n} 1}\right)\right),\right. \\
\left.\left(\left(x_{i}, v_{j}\right),\left(y_{i}, v_{j+{ }_{n} 1}\right)\right)\right\}_{i=0, \ldots, m-1}^{j=0, \ldots, n-1} .
\end{gathered}
$$

By Theorem 1.3, the digraph induced by the vertices of the form $\left(x_{i}, v_{j}\right)$ is a cycle of length $m n$ with a strong orientation. By the definition of the Kronecker product, we have $m n$ spokes of the form $\left(\left(x_{i}, v_{j}\right),\left(y_{i}, v_{j+{ }_{n} 1}\right)\right)$ and inner edges of the form $\left(\left(y_{i}, v_{j}\right),\left(y_{i+{ }_{m} k}, v_{j+{ }_{n} 1}\right)\right)$. Let us see now that $d\left(\left(x_{i}, v_{j-{ }_{n} 1}\right),\left(x_{i+{ }_{m} k}, v_{j}\right)\right)=$ $k+m r$, where $r$ is the smallest positive integer such that $k+{ }_{n} m r=1$. By the definition of $\overrightarrow{P(m ; k)}$ there is a directed path of length $k$ from $x_{i}$ to $x_{i+k}=k$. Thus $d\left(\left(x_{i}, v_{j}\right),\left(x_{i}, v_{j+{ }_{n} m}\right)\right)=m$ and hence

$$
\begin{aligned}
d\left(\left(x_{i}, v_{j-1}\right),\left(x_{i+k}, v_{j}\right)\right)= & d\left(\left(x_{i}, v_{j-1}\right),\left(x_{i+k}, v_{j-1+k}\right)\right) \\
& +d\left(\left(x_{i+k}, v_{j-1+k}\right),\left(x_{i+k}, v_{j}\right)\right) \\
= & k+d\left(\left(x_{i+k}, v_{j-m r}\right),\left(x_{i+k}, v_{j}\right)\right)=k+m r .
\end{aligned}
$$

This completes the proof.

2.1. (Super) edge-magic GGP. Since every digraph $\overrightarrow{L P_{m}}$ admits a super edgemagic labeling (just label the vertices of the path following the arrows in increasing order) we can apply Theorem 1.2 to extend the class of graphs that are super edgemagic, by adding every GGP that can be obtained from the $\otimes_{h}$-product of the $\overrightarrow{L P_{m}}$ with $S_{n}$. For instance, next we propose an alternative proof for the following theorem found in $[6,7]$.

TheOREM $2.5[6,7]$. Let $m, n$ be two integers, $n$ odd. Then $P_{m} \times C_{n}$ is super edgemagic.

PROOF. Since, by Theorem 1.2 $\overrightarrow{L P_{m}} \otimes \overrightarrow{C_{n}}$ is super edge-magic and by Proposition 2.1 und $\left(\overrightarrow{L P_{m}} \otimes \overrightarrow{C_{n}}\right)=P_{m} \times C_{n}$, the result follows.

THEOREM 2.6. The Petersen graph is super edge-magic. Moreover we have the following results.

(i) For each $m \geq 2,1<l \leq m$ and $1 \leq k \leq 2$, the graph $\operatorname{GGP}\left(5 ; \pi_{2}, \ldots, \pi_{m}\right)$, where $\pi_{i}=\sigma_{1}$ for $i \neq l$ and $\pi_{l}=\sigma_{k}$, is super edge-magic.

(ii) For each $1 \leq k \leq 2$, the graph $P(5 n ; k+5 r)$ is super edge-magic, where $r$ is the smallest positive integer such that $k+{ }_{n} 5 r=1$. 
PROOF. Let $a_{0} a_{1}$ be a directed path in $\overrightarrow{L P_{2}}$. Let $\vec{C}_{5}$ be the directed cycle defined by $1 \rightarrow 4 \rightarrow 2 \rightarrow 5 \rightarrow 3 \rightarrow 1$ and $\overrightarrow{C_{1}} \cup \overrightarrow{C_{4}}$ the digraph $1 \rightarrow 5 \rightarrow 3 \rightarrow 4 \rightarrow 1$ with a loop labeled 2. We can obtain the Petersen graph from $\overrightarrow{L P_{2}} \otimes_{h}\left\{\overrightarrow{C_{5}}, \overrightarrow{C_{1}} \cup \overrightarrow{C_{4}}\right\}$, where $h$ is defined by $h\left(a_{0} a_{0}\right)=h\left(a_{1} a_{1}\right)=\overrightarrow{C_{5}}$ and $h\left(a_{0} a_{1}\right)=\overrightarrow{C_{1}} \cup \overrightarrow{C_{4}}$. By Theorem $2.5 P(5 ; 1)$ is super edge-magic. Thus, applying Proposition 2.3 together with Theorem 1.2, we obtain (i). Similarly, by Proposition 2.4 and Theorem 1.2 we obtain (ii).

\section{Edge bi-magic}

A $(p, q)$-graph $G=(V, E)$ is said to have an edge bi-magic labeling if there exists a bijective function $f: V \cup E \longrightarrow\{i\}_{i=1}^{p+q}$ such that for each edge $u v \in E$, $f(u)+f(u v)+f(v) \in\left\{k_{1}, k_{2}\right\}$, where $k_{1}, k_{2}$ are two distinct constants. In this case, the graph is said to be edge bi-magic. If we add the extra condition that $f(V)=\{i\}_{i=1}^{p}$ then we say that $f$ is a super edge bi-magic labeling and $G$ a super edge bi-magic graph. In this section, we study the complete graphs that are edge bi-magic and we introduce new classes of (super) edge bi-magic graphs. In particular, we generalize the class of edge bi-magic graphs that was given by Rajan et al. in [11]. We also prove that the product introduced in [8] is useful for providing new families of edge bi-magic graphs.

The next theorem gives necessary conditions for a complete graph to be edge bimagic, provided that the magic constants are of the same parity. It is similar to [13, Theorem 2.11]. See also [12].

THEOREM 3.1. Suppose that $K_{p}$ has an edge bi-magic labeling with magic constants $k_{1}, k_{2}$ such that $k_{1}+k_{2}$ is an even integer. The number $v$ of vertices that receive even labels satisfies the following condition.

(i) If $p \equiv 0$ or $3(\bmod 4)$ and $k_{1}$ is even then $v=\frac{1}{2}(p-1 \pm \sqrt{p+1})$.

(ii) If $p \equiv 1$ or $2(\bmod 4)$ and $k_{1}$ is even then $v=\frac{1}{2}(p-1 \pm \sqrt{p-1})$.

(iii) If $p \equiv 0$ or $3(\bmod 4)$ and $k_{1}$ is odd then $v=\frac{1}{2}(p+1 \pm \sqrt{p+1})$.

(iv) If $p \equiv 1$ or $2(\bmod 4)$ and $k_{1}$ is odd then $v=\frac{1}{2}(p+1 \pm \sqrt{p+1})$.

PROOF. The proof is similar to the one given [13, Theorem 2.11]. It is only relevant to note that $k_{1}$ and $k_{2}$ are of the same parity.

LEMMA 3.2. Let $G$ be a super edge bi-magic graph of order $p>4$ without loops. Then its size is at most $4 p-10$.

PROOF. Let $G$ be a super edge bi-magic graph of order $p>4$ without loops and let $f$ be a super edge bi-magic labeling of $G$. Consider the set $S_{E}=\{f(u)+f(v): u v \in$ $E(G)\}$. Then if we allow repetitions in $S_{E}$ we have that

$$
S_{E} \subset\{3,4, \ldots, 2 p-1\} \cup\{5, \ldots, 2 p-3\} .
$$

Therefore, the size of a super edge bi-magic graph without loops is at most $4 p-10$. 


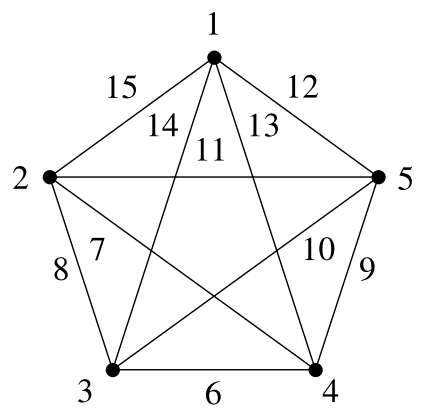

FIGURE 3. A super edge bi-magic labeling of $K_{5}$.

REMARK 3.3. This upper bound is tight. Figure 3 shows an edge bi-magic labeling of $K_{5}$. Using Lemma 3.2 we obtain that the graph $K_{n}$ is not super edge bi-magic for $n>5$.

The next lemma gives a characterization of super edge bi-magic graphs in terms of arithmetic progressions. In some sense, it is a similar result to Lemma 1.1 for the case of super edge-magic labelings given by Figueroa et al. in [7].

LEMMA 3.4. A graph labeling of $G$ is super edge bi-magic if and only if the set of sum labels of adjacent vertices (including repetitions) can be partitioned into two sets $S$ and $S^{\prime}$ and there exists an integer $r$ such that $S \cup\left(S^{\prime}-r\right)$ is a set of consecutive integers.

PROOF. In order to prove the necessity assume that there exists a super edge bimagic labeling of $G$. Let $k$ and $k^{\prime}$ be the two magic constants and let $S$ (respectively $S^{\prime}$ ) be the sums of the labels of adjacent vertices with magic sum $k$ (respectively $\left.k^{\prime}\right)$. Thus $(k-S) \cup\left(k^{\prime}-S^{\prime}\right)$ forms a set of consecutive integers (the labels of the edges). Hence, so do the sets $(S-k) \cup\left(S^{\prime}-k^{\prime}\right)$ and $S \cup\left(S^{\prime}-\left(k^{\prime}-k\right)\right)$. Let us prove the converse. Let $S \cup\left(S^{\prime}-r\right)=\left\{a_{1}<\cdots<a_{q}\right\}$ and assume first that $a_{1} \in S$. We have that $a_{i}+p+q-i+1=k$ is constant. For each $1 \leq i \leq q$ we assign to the corresponding edge the label $p+q-i+1$. Thus, for each $a_{i} \in S$ we have $a_{i}+p+q-i+1=k$, whereas if $a_{i} \in S^{\prime}-r$ we obtain that $a_{i}+r+p+q-$ $i+1=k+r=k^{\prime}$. We proceed similarly in case $a_{1}+r \in S^{\prime}$.

3.1. Some constructions of (super) edge bi-magic graphs. Let $G=(V, E)$ be a graph and let $S \subset V$. We denote by $G *_{S} u$ the graph obtained from $G$ by adding a new vertex $u$ and the edge set $\{u v: v \in S\}$ and by $G \wedge_{S}\left\{u_{i}\right\}_{i=1}^{|S|}$ the graph obtained from $G$ by adding a leaf $v_{i} u_{i}$ to each vertex of $v_{i} \in S$. Furthermore, in general we write $G \wedge_{S}\left\{u_{i}^{j}\right\}_{i=1, \ldots,|S|}^{j=1, \ldots, n_{i}}$ to denote the graph obtained from $G$ by adding leaves $v_{i} u_{i}^{j}$, $j=1, \ldots, n_{i}$ to each vertex of $v_{i} \in S$. 
Proposition 3.5. Let $G=(V, E)$ be a $(p, q)$-graph with a (super) edge-magic labeling $f$. Let $S \subset V$ be a subset of vertices such that $\{f(v)\}_{v \in S}$ is a set of consecutive integers. Then the graph $G *_{s} u$ is (super) edge bi-magic.

Proof. Let $G * S u=\left(V^{\prime}, E^{\prime}\right)$ and assume that $s=\max \{f(x) \mid x \in S\}$. We consider the labeling $f^{\prime}: V^{\prime} \cup E^{\prime} \longrightarrow\{i\}_{i=1}^{p+q+|S|+1}$ such that

$$
f^{\prime}(x)= \begin{cases}f(x)+1 & \text { if } x \in V \cup E, \\ 1 & \text { if } x=u, \\ p+q+2+i & \text { if } x=u v, v \in S, \text { and } f(v)=s-i .\end{cases}
$$

Then $f^{\prime}$ is a (super) edge bi-magic labeling of $G *_{S} u=\left(V^{\prime}, E^{\prime}\right)$ with magic constants $k_{1}=k+3$ and $k_{2}=p+q+s+4$, where $k$ is the magic sum for $f$.

The graph $P Y(n)$ is the graph obtained from the cylinder $C_{3} \times P_{n}$ by adding a new vertex and joining it to the three vertices of the cycle on the top.

COROLlary 3.6 [11, Theorem 1]. The graph PY $(n)$ is edge bi-magic.

PROOF. Recall that und $\left(\overrightarrow{L P_{n}} \otimes \overrightarrow{C_{3}}\right)=C_{3} \times P_{n}$. In particular, it admits a (super) edge-magic labeling, with the vertices of the cycle on the top labeled with $\{1,2,3\}$. Thus, the construction of Proposition 3.5 produces an edge bi-magic labeling of $P Y(n)$.

Proposition 3.7. Let $G=(V, E)$ be a $(p, q)$-graph with a (super) edge-magic labeling $f$. Let $S$ be a subset of vertices such that $\{f(v)\}_{v \in S}$ is a set of consecutive integers and $|S|$ is odd. Then the graph $G \wedge_{S}\left\{u_{i}\right\}_{i=1}^{|S|}$ is (super) edge bi-magic.

Proof. Let $G \wedge_{S}\left\{u_{i}\right\}_{i=1}^{|S|}=\left(V^{\prime}, E^{\prime}\right)$ and assume that $s=\max \{f(x) \mid x \in S\}$ and that the new edges are $v_{i} u_{i}$ where $f\left(v_{i}\right)=s-i+1$. We consider the labeling $f^{\prime}: V^{\prime} \cup E^{\prime} \longrightarrow\{i\}_{i=1}^{p+q+|S|+1}$ such that

$$
f^{\prime}(x)= \begin{cases}f(x)+|S| & \text { if } x \in V \cup E, \\ \frac{|S|-1}{2}+\frac{i+1}{2} & \text { if } x=u_{i} \text { and } i \text { is odd, } \\ \frac{2}{p} & \text { if } x=u_{i} \text { and } i \text { is even, } \\ p+q+|S|+l & \text { if } x=v_{i} u_{i} \text { and } i=2 l-1, \\ p+q+|S|+\frac{|S|+1}{2}+l & \text { if } x=v_{i} u_{i} \text { and } i=2 l\end{cases}
$$

Then $f^{\prime}$ is a (super) edge bi-magic labeling of $G \wedge_{S}\left\{u_{i}\right\}_{i=1}^{|S|}$ with magic constants $k_{1}=k+3|S|$ and $k_{2}=p+q+s+(5|S|+3 l) / 2$, where $k$ is the magic sum of $f$. 
Proposition 3.8. Let $G=(V, E)$ be a $(p, q)$-graph with a (super) edge-magic labeling $f$. Let $S$ be a subset of vertices such that $f\left(v_{i}\right)=s-d(i-1)$ for each $v_{i} \in S$ with $d>1$. Then the graph $G \wedge S\left\{u_{i}^{j}\right\}_{i=1, \ldots,|S|}^{j=1, \ldots, n_{i}}$, where $n_{2 l-1}=d-1$ and $n_{2 l}=1$, is (super) edge bi-magic.

Proof. Let $G \wedge_{S}\left\{u_{i}^{j}\right\}_{i=1, \ldots,|S|}^{j=1, \ldots, n_{i}}=\left(V^{\prime}, E^{\prime}\right)$. Let $r=(d-1)\lceil|S| / 2\rceil+\lfloor|S| / 2\rfloor$. We consider the labeling $f^{\prime}: V^{\prime} \cup E^{\prime} \longrightarrow\{i\}_{i=1}^{p+q+2 r}$ such that

$$
f^{\prime}(x)= \begin{cases}f(x)+r & \text { if } x \in V \cup E, \\ (l-1) d+j & \text { if } x=u_{2 l-1}^{j}, \\ l d & \text { if } x=u_{2 l}^{1}, \\ p+q+r+l d-j & \text { if } x=v_{2 l-1} u_{2 l-1}^{j}, \\ p+q+r+l d & \text { if } x=v_{2 l} u_{2 l}^{1} .\end{cases}
$$

Then $f^{\prime}$ is a (super) edge bi-magic labeling of $G \wedge S\left\{u_{i}^{j}\right\}_{i=1, \ldots,|S|}^{j=1, \ldots, n_{i}}$ with magic constants $k_{1}=k+3 r$ and $k_{2}=p+q+d+2 r+s$, where $k$ is the magic sum of $f$.

3.2. (Super) edge bi-magic graphs obtained using $\otimes_{h}$-product. We present a simplified proof of the main result found in [8]. Recall that $S_{n}$ denotes the set of all super edge-magic 1-regular labeled digraphs of odd order $n$.

Theorem 3.9. Let $D$ be a (super) edge-magic digraph and let $h: E(D) \rightarrow S_{n}$ be any function. Then the graph und $\left(D \otimes_{h} S_{n}\right)$ is (super) edge-magic.

PROOF. As in the original paper, we rename the vertices of $D$ and each element of $S_{n}$ after the labels of their corresponding edge-magic and super edge-magic labelings respectively. We also define the labels as in [8, Theorem 3.1].

(1) If $(i, j) \in V\left(D \otimes_{h} S_{n}\right)$ we assign to the vertex the label: $n(i-1)+j$.

(2) If $\left((i, j),\left(i^{\prime}, j^{\prime}\right)\right) \in E\left(D \otimes_{h} S_{n}\right)$ we assign to the arc the label: $n(e-1)+$ $(3 n+3) / 2-\left(j+j^{\prime}\right)$, where $e$ is the label of $\left(i, i^{\prime}\right)$ in $D$.

Notice that, since each element $\Gamma$ of $S_{n}$ is labeled with a super edge-magic labeling, by [8, Corollary 1.1$]$ we have

$$
\left\{(3 n+3) / 2-\left(j+j^{\prime}\right):\left(j, j^{\prime}\right) \in E(\Gamma)\right\}=\{1,2, \ldots, n\} .
$$

Thus, the set of labels in $D \otimes_{h} S_{n}$ covers all elements in $\{1,2, \ldots, n(|V(D)|+$ $|E(D)|)\}$. Moreover, for each arc $\left((i, j)\left(i^{\prime}, j^{\prime}\right)\right) \in E\left(D \otimes_{h} S_{n}\right)$, coming from an arc $e=\left(i, i^{\prime}\right) \in E(D)$ and an arc $\left(j, j^{\prime}\right) \in E\left(h\left(i, i^{\prime}\right)\right)$, the sum of labels is constant and equal to

$$
n\left(i+i^{\prime}+e-3\right)+(3 n+3) / 2 .
$$

That is, $n\left(\operatorname{val}_{f}-3\right)+(3 n+3) / 2$. We also notice that if the digraph $D$ is super edge-magic then the vertices of $D \otimes_{h} S_{n}$ receive the smallest labels. 
Using this proof we can extend the previous result to the case of edge bi-magic digraphs.

THEOREM 3.10. Let $D$ be a (super) edge bi-magic digraph and let $h: E(D) \rightarrow S_{n}$ be any function. Then the graph und $\left(D \otimes_{h} S_{n}\right)$ is (super) edge bi-magic.

PROOF. Let $k_{1}$ and $k_{2}$ be the valences for a (super) edge bi-magic labeling of $D$. From the proof of Theorem 1.2, it is clear that for each arc $\left((i, j),\left(i^{\prime}, j^{\prime}\right)\right) \in E\left(D \otimes_{h} S_{n}\right)$, coming from an arc $\left(i, i^{\prime}\right)$ in $D$ labeled with $e$, the induced sum (3.1) belongs to $\left\{n\left(k_{1}-3\right)+(3 n+3) / 2, n\left(k_{2}-3\right)+(3 n+3) / 2\right\}$.

\section{4. $k$-equitable}

In this section, we use the $\otimes_{h}$-product in order to construct $k$-equitable labelings of new families of graphs. In this case, the input elements are $k$-equitable digraphs and 1-regular super edge-magic digraphs. However, instead of applying the product directly, we have to use what we call the rotation of a super edge-magic digraph.

4.1. Rotations of super edge-magic digraphs. Let $M=\left(a_{i, j}\right)$ be a square matrix of order $n$ and let $M^{R}=\left(a_{i, j}^{R}\right)$ be the matrix obtained from $M$ where $a_{i, j}^{R}=a_{n+1-j, i}$. Graphically this corresponds to a rotation of the matrix by $\pi / 2$ radians clockwise (see Example 4.1). We say that $M^{R}$ is the rotation of the matrix $M$. Note that the digraph corresponding to $M^{R}$ may contain loops and double arcs. Therefore, in this section we may work with digraphs for which their underlying graphs contain multiple edges. Recall that if we write $S_{n}$ then $n$ is odd.

EXAMPLE 4.1. A matrix $M$ and its rotation $M^{R}$

$$
M=\left(\begin{array}{lll}
0 & 1 & 0 \\
0 & 0 & 1 \\
1 & 0 & 0
\end{array}\right) \rightarrow M^{R}=\left(\begin{array}{lll}
1 & 0 & 0 \\
0 & 0 & 1 \\
0 & 1 & 0
\end{array}\right)
$$

LEMMA 4.2. Let $D \in S_{n}$, and assume that each vertex is named after the label of a super edge-magic labeling. Let $A=\left(a_{i, j}\right)$ be its adjacency matrix. If $a_{i, j}^{R}=1$ then

$$
|i-j| \leq \frac{n-1}{2} .
$$

ProOF. By [8, Corollary 1.1], if $A=\left(a_{i, j}\right)$ is the adjacency matrix of $D \in S_{n}$ and $a_{i, j}=1$ then $(n+3) / 2 \leq i+j \leq(3 n+1) / 2$. Hence, since $a_{i, j}^{R}=a_{n+1-j, i}$, if $a_{i, j}^{R}=$ 1 it follows that $(n+3) / 2 \leq n+1-j+i \leq(3 n+1) / 2$. Therefore, $-(n-1) / 2 \leq$ $i-j \leq(n-1) / 2$ and we obtain the result.

A digraph $S$ is said to be a rotation super edge-magic of order $n$ if its adjacency matrix is the rotation matrix of the adjacency matrix of a super edge-magic 1-regular digraph of order $n$. We denote by $R S_{n}$ the set of all digraphs that are rotation super edge-magic of order $n$. The following corollaries are easy observations. 
COROllary 4.3. Let $S$ be a digraph in $R S_{n}$ and let $k$ be an integer. If $|k| \leq$ $(n-1) / 2$ then there exists a unique arc $(i, j) \in E(S)$ such that $i-j=k$.

Proof. Let $D \in S_{n}$ be the digraph where $S$ is coming from. Let $A=\left(a_{i, j}\right)$ be the adjacency matrix of $D$, where every vertex takes the label of a super edge-magic labeling of $D$. Note that, since $A$ comes from a super edge-magic labeling of a 1-regular digraph, every secondary diagonal $(\nearrow)$ contains at most a 1 , and the diagonals that contain the $1 \mathrm{~s}$ are consecutive. Moreover, in each main diagonal ( $\searrow$ ) of $A^{R}$ there appears at most a 1 and the diagonals that contain the $1 \mathrm{~s}$ are consecutive.

Corollary 4.4. For each digraph $D$ and each constant function $h: E(D) \longrightarrow R S_{n}$ one of the weakly connected components of $D \otimes_{h} R S_{n}$ is isomorphic to $D$.

Proof. Let $S$ be a digraph in $R S_{n}$. By Corollary 4.3 we know that $S$ contains a loop. Let $(j, j)$ be a loop in $S$. Then the subdigraph of $D \otimes_{h} R S_{n}$ induced by the vertices of the form $(i, j)$ for $i \in V(D)$ is isomorphic to $D$.

REMARK 4.5. Inheriting the notation used in this section, let $A$ be the adjacency matrix of a super edge-magic digraph $D$ of order $n$. We have that, $A^{R}=A^{t} P$, where $A^{t}$ is the transpose of $A$, and $P=\left(p_{i, j}\right)$ where $p_{i, j}=1$ if $i+j=n+1$ but $p_{i, j}=0$ otherwise. Clearly, $\left(A^{R}\right)^{t}$ is the adjacency matrix of some digraph in $R S_{n}$. That is, there exists a (possibly) different super edge-magic labeling of $D$, such that if $B$ is its induced adjacency matrix then $B^{t} P=\left(A^{R}\right)^{t}$. Thus, $B=P A^{t} P$.

EXAMPLE 4.6. Let $D$ be the super edge-magic digraph $1 \rightarrow 5 \rightarrow 3 \rightarrow 4 \rightarrow 1$ and a loop in 2. Its adjacency matrix $A$ is

$$
A=\left(\begin{array}{lllll}
0 & 0 & 0 & 0 & 1 \\
0 & 1 & 0 & 0 & 0 \\
0 & 0 & 0 & 1 & 0 \\
1 & 0 & 0 & 0 & 0 \\
0 & 0 & 1 & 0 & 0
\end{array}\right)
$$

which has rotation matrix

$$
A^{R}=\left(\begin{array}{lllll}
0 & 1 & 0 & 0 & 0 \\
0 & 0 & 0 & 1 & 0 \\
1 & 0 & 0 & 0 & 0 \\
0 & 0 & 1 & 0 & 0 \\
0 & 0 & 0 & 0 & 1
\end{array}\right)
$$

Then $\left(A^{R}\right)^{t}=B^{t} P$ where $B=P A^{t} P$. That is, $B$ is the adjacency matrix of a super edge-magic digraph obtained by reversing the arcs of $D$ and by interchanging the labels by $\sigma$, where $\sigma$ is the permutation on $\{1, \ldots, n\}$ defined by $\sigma(i)=n+1-i$. In our example, the super edge-magic digraph defined by $B$ is $1 \rightarrow 5 \rightarrow 2 \rightarrow 3 \rightarrow 1$ and the loop in 4. 
REMARK 4.7. Let $M^{3 R}$ be the matrix obtained from $M$ by rotating $3 \pi / 2$ radians in the clockwise sense the columns of $M$. That is, $M^{3 R}=P A^{t}$. Note that this different rotation of the adjacency matrix of a super edge-magic labeled digraph has the same properties of $M^{R}$.

4.2. Main theorem. Let $D$ be a $k$-equitable digraph where the vertices are identified by the labels of a $k$-equitable labeling of $D$. Let us consider the induced labeling on $V\left(G \otimes_{h} R S_{n}\right)$ that assigns the label $n(i-1)+j$ to the vertex $(i, j)$. One can easily see that all labels are distinct and that, in case the labeling of $D$ is optimal, all elements in $\{1, \ldots, n \cdot|V(D)|\}$ are used. Moreover, by the product definition of $\otimes_{h},\left|n\left(i-i^{\prime}\right)+\left(j-j^{\prime}\right)\right|$ is an induced arc label if and only if $\left(i, i^{\prime}\right) \in E(D)$ and $\left(j, j^{\prime}\right) \in E\left(h\left(i, i^{\prime}\right)\right)$.

LEMMA 4.8. Let D be a k-equitable digraph, and let $\left((i, j),\left(i^{\prime}, j^{\prime}\right)\right),\left((r, s),\left(r^{\prime}, s^{\prime}\right)\right)$ be two arcs of $D \otimes_{h} R S_{n}$. If $\left|n\left(i-i^{\prime}\right)+\left(j-j^{\prime}\right)\right|=\left|n\left(r-r^{\prime}\right)+\left(s-s^{\prime}\right)\right|$ then $\left|i-i^{\prime}\right|=\left|r-r^{\prime}\right|$ and $\left|s-s^{\prime}\right|=\left|j-j^{\prime}\right|$.

PROOF. Note that the equality $n\left(i-i^{\prime}\right)+\left(j-j^{\prime}\right)= \pm\left(n\left(r-r^{\prime}\right)+\left(s-s^{\prime}\right)\right)$ implies that there exists $\alpha \in \mathbb{Z}$ such that $|\alpha n|=\left| \pm\left(s-s^{\prime}\right)-\left(j-j^{\prime}\right)\right|$. Thus, by Lemma 4.2, $|\alpha n| \leq n-1$. Hence, $\alpha=0$ and therefore $\left|j-j^{\prime}\right|=\left|s-s^{\prime}\right|$ and $\left|i-i^{\prime}\right|=\left|r-r^{\prime}\right|$.

THEOREM 4.9. Let $D$ be an (optimal) k-equitable digraph and let $h: E(D) \rightarrow R S_{n}$ be any function. Then $D \otimes_{h} R S_{n}$ is (optimal) $k$-equitable.

ProOf. Assume that $\left|n\left(i-i^{\prime}\right)+\left(j-j^{\prime}\right)\right|$ is an arc label induced by a $k$-equitable labeling of $D$. There exist exactly $k$ arcs in $D,\left(i_{l}, i_{l}^{\prime}\right), 1 \leq l \leq k$ such that $\left|i_{l}-i_{l}^{\prime}\right|=$ $\left|i-i^{\prime}\right|$. Thus $\left|n\left(i_{l}-i_{l}^{\prime}\right)\right|=\left|n\left(i-i^{\prime}\right)\right|$ and by Lemma 4.2 we have that

$$
\left|n\left(i_{l}-i_{l}^{\prime}\right)\right|-\frac{n-1}{2} \leq\left|n\left(i-i^{\prime}\right)+\left(j-j^{\prime}\right)\right| \leq\left|n\left(i_{l}-i_{l}^{\prime}\right)\right|+\frac{n-1}{2} .
$$

Hence, we obtain that

$$
|| n\left(i-i^{\prime}\right)+\left(j-j^{\prime}\right)|-| n\left(i_{l}-i_{l}^{\prime}\right)|| \leq \frac{n-1}{2}
$$

and by Corollary 4.3 there exist two different $\operatorname{arcs}\left(r, r^{\prime}\right),\left(s, s^{\prime}\right) \in E\left(h\left(i_{l}, i_{l}^{\prime}\right)\right)$ such that

$$
|| n\left(i-i^{\prime}\right)+\left(j-j^{\prime}\right)|-| n\left(i_{l}-i_{l}^{\prime}\right)||=\left|r-r^{\prime}\right|=\left|s-s^{\prime}\right|
$$

with $r-r^{\prime} \leq 0 \leq s-s^{\prime}$.

Therefore, either $\left|n\left(i-i^{\prime}\right)+\left(j-j^{\prime}\right)\right|=\left|n\left(i_{l}-i_{l}^{\prime}\right)+r-r^{\prime}\right|$ or $\mid n\left(i-i^{\prime}\right)+$ $\left(j-j^{\prime}\right)|=| n\left(i_{l}-i_{l}^{\prime}\right)+s-s^{\prime} \mid$. In the first case $\left(\left(i_{l}, r\right),\left(i_{l}^{\prime}, r^{\prime}\right)\right)$ is labeled with $\left|n\left(i-i^{\prime}\right)+\left(j-j^{\prime}\right)\right|$, whereas in the second case $\left(\left(i_{l}, s\right),\left(i_{l}^{\prime}, s^{\prime}\right)\right)$ is labeled with $\left|n\left(i-i^{\prime}\right)+\left(j-j^{\prime}\right)\right|$.

Moreover, assume that

$$
\left|n\left(i-i^{\prime}\right)+\left(j-j^{\prime}\right)\right|=\left|n\left(r-r^{\prime}\right)+\left(s-s^{\prime}\right)\right| .
$$


By Lemma 4.8, $\left|i-i^{\prime}\right|=\left|r-r^{\prime}\right|$ and $\left|s-s^{\prime}\right|=\left|j-j^{\prime}\right|$. That is, $\left|n\left(i-i^{\prime}\right)\right|=$ $\left|n\left(r-r^{\prime}\right)\right|$ and we only have $k$-possible arcs with the same label.

In particular, if the $k$-equitable labeling of $D$ is optimal, then the induced labeling on $D \otimes_{h} R S_{n}$ is also optimal.

Recall that cycles are $k$-equitable for each proper divisor $k$ of their size. By giving a nonoptimal labeling, it was stated in [3] that the union of vertex-disjoint $k$-equitable graphs is $k$-equitable. Using Theorem 4.9 , we can provide optimal $k$-equitable labelings of $n$ copies of trees, for $n$ odd.

THEOREM 4.10. Let $n$ be an odd integer and let $F$ be an optimal $k$-equitable forest for each proper divisor $k$ of $|E(F)|$. Then $n F$ is optimal $k$-equitable for each proper divisor $k$ of $|E(F)|$.

PROOF. Clearly, each rotation of a super edge-magic 1-regular digraph gives a 1-regular digraph. In particular, by Theorem 1.4 we have that und $\left(\vec{F} \otimes_{h} \Sigma_{n}\right)=n F$. Thus, since $F$ is optimal $k$-equitable for each proper divisor $k$ of $|E(F)|$, Theorem 4.9 implies that $n F$ is optimal $k$-equitable for each proper divisor $k$ of $|E(F)|$.

THEOREM 4.11. Let $m-1, n$ be odd integers. Then $n C_{m}$ is optimal $k$-equitable for all proper divisors $k$ of $m$.

PROOF. Let $\overrightarrow{C_{n}}$ be a strong orientation of $C_{n}$ and assume that $M$ is the adjacency matrix of $\vec{C}_{n}$ where each vertex is identified with the label of a super edge-magic labeling of $\overrightarrow{C_{n}}$. The matrix $M^{R}$ obtained by rotating $\pi / 2$ radians clockwise is the adjacency matrix of a digraph $\overrightarrow{R C_{n}}=\overrightarrow{C_{1}} \cup \overrightarrow{C_{n_{1}}} \cup \cdots \cup \overrightarrow{C_{n_{k}}}$. Let $\overleftarrow{R C_{n}}$ be the digraph obtained from $\overrightarrow{R C_{n}}$ by reversing all its arcs. Consider a function $h$ : $E\left(\overrightarrow{C_{m}}\right) \rightarrow\left\{\overrightarrow{R C_{n}}, \overleftarrow{R C_{n}}\right\}$ such that two consecutive arcs in $\overrightarrow{C_{m}}$, namely $(x, y),(y, z)$ have $h(x, y) \neq h(y, z)$. Assume that $a_{1} a_{2} \cdots a_{m}$ is a directed path in $\overrightarrow{C_{m}}$. Then for each $(i, j) \in E\left(h\left(a_{1}, a_{2}\right)\right)$ we obtain that $\left(a_{1}, i\right)\left(a_{2}, j\right)\left(a_{3}, i\right) \cdots\left(a_{m}, j\right)\left(a_{1}, i\right)$ is a cycle of length $m$ in $\overrightarrow{C_{m}} \otimes_{h}\left\{\overrightarrow{R C_{n}}, \overleftarrow{R C_{n}}\right\}$. That is,

$$
\overrightarrow{C_{m}} \otimes_{h}\left\{\overrightarrow{R C_{n}}, \overleftarrow{R C_{n}}\right\} \simeq n \overrightarrow{C_{m}}
$$

Thus, since every cycle is optimal $k$-equitable for each proper divisor $k$ of the size, the result follows by Theorem 4.9 .

\section{5. (Super) edge $r$-magic graphs. Open problems}

A $(p, q)$-graph $G=(V, E)$ admits an edge $r$-magic labeling if there exists a bijective function $f: V \cup E \longrightarrow\{i\}_{i=1}^{p+q}$ such that for each edge $u v \in E, f(u)+$ $f(u v)+f(v) \in\left\{k_{1}, k_{2}, \ldots, k_{r}\right\}$ where $\left\{k_{1}, \ldots, k_{r}\right\}$ are $r$ distinct constants. In this case, the graph is said to be edge $r$-magic. If we add the extra condition 
that $f(V)=\{i\}_{i=1}^{p}$ then we say that $f$ is a super edge $r$-magic labeling and $G$ a super edge r-magic graph.

The next lemma is an extension of Lemma 3.4 for the case of super edge $r$-magic graphs. The proof works similarly.

LEMMA 5.1. A graph labeling of a graph $G$ is super edge $r$-magic if and only if the set of sum labels of adjacent vertices (including repetitions) can be partitioned into $r$ sets $S_{0}, S_{1}, \ldots, S_{r-1}$ and there exist $r-1$ integers $c_{1}, c_{2}, \ldots, c_{r-1}$ such that $S_{0} \cup\left(S_{1}-c_{1}\right) \cup \cdots \cup\left(S_{r-1}-c_{r-1}\right)$ is a set of consecutive integers.

With a similar proof as in Section 3.2 we can state the following result.

THEOREM 5.2. Let $D$ be a (super) edge $r$-magic digraph and let $h: E(D) \rightarrow S_{n}$. Then the graph und $\left(D \otimes_{h} S_{n}\right)$ is (super) edge $r$-magic.

Clearly, each graph is edge $r$-magic for some $r$. Thus a natural question appears.

Question 5.3. Given a graph $G$, find the minimum $r$ such that $G$ is edge $r$-magic.

Similarly, we can study the following aspect.

QUESTION 5.4. Let $G$ be an edge $r$-magic graph. Find an edge $r$-magic labeling $f$ of $G$ that minimizes the difference $k_{r}-k_{1}$, where $k_{1}$ and $k_{r}$ are, respectively, the minimum and the maximum magic constants of $f$.

\section{References}

[1] B. D. Acharya and S. M. Hegde, 'Strongly indexable graphs', Discrete Math. 93 (1991), 123-129.

[2] C. Barrientos, 'Difference vertex labelings', PhD Thesis, Universitat Politècnica de Catalunya, 2004.

[3] C. Barrientos, I. J. Dejter and H. Hevia, 'Equitable labelings of forests', in: Combinatorics and Graph Theory'95, Vol.1 (ed. Y. Alavi) (World Scientific, Singapore, 1995), pp. 1-26.

[4] G. Bloom and S. Ruiz, 'Decomposition into linear forest and difference labelings of graphs', Discrete Appl. Math. 49 (1994), 13-37.

[5] G. Chartrand and L. Lesniak, Graphs and Digraphs, 2nd edn (Wadsworth \& Brooks/Cole Advanced Books and Software, Monterey, 1986).

[6] H. Enomoto, A. Lladó, T. Nakamigawa and G. Ringel, 'Super edge-magic graphs', SUT J. Math. 34 (1998), 105-109.

[7] R. M. Figueroa-Centeno, R. Ichishima and F. A. Muntaner-Batle, 'The place of super edge-magic labelings among other classes of labelings', Discrete Math. 231(1-3) (2001), 153-168.

[8] R. M. Figueroa-Centeno, R. Ichishima, F. A. Muntaner-Batle and M. Rius-Font, 'Labeling generating matrices', J. Combin. Math. Combin. Comput. 67 (2008), 189-216.

[9] J. A. Gallian, 'A dynamic survey of graph labeling', Electron. J. Combin. 17 (2010), \#DS6.

[10] A. Kotzig and A. Rosa, 'Magic valuations of finite graphs', Canad. Math. Bull. 13 (1970), 451-461.

[11] B. Rajan, I. Rajasingh and M. A. Basker, 'Edge bi-magic total labelings of graphs', Proc. 5th Asian Mathematical Conf., Malaysia, June 2009 (eds. L. S. Keong and H. K. Haith) (June 2009), pp. 426-428.

[12] L. Valdés, 'Edge-magic $K_{p}$ '. Paper delivered at Thirty-Second South Eastern International Conference on Combinatorics, Graph Theory and Computing, Baton Rouge, 2001.

[13] W. D. Wallis, Magic Graphs (Birkhäuser, Boston, MA, 2001). 
[14] D. B. West, Introduction to Graph Theory (Prentice Hall, Upper Saddle River, NJ, 1996).

[15] J. Wojciechowski, 'Long induced cycles in the hypercube and colourings of graphs', PhD Thesis, University of Cambridge, 1990.

[16] J. Wojciechowski, 'Equitable labelings of cycles’, J. Graph Theory 17 (1993), 531-547.

S. C. LÓPEZ, Departament de Matemàtica Aplicada IV,

Universitat Politècnica de Catalunya, C/Esteve Terrades 5, 08860 Castelldefels, Spain

e-mail: susana@ma4.upc.edu

F. A. MUNTANER-BATLE, Graph Theory and Applications Research Group, School of Electrical Engineering and Computer Science,

Faculty of Engineering and Built Environment, The University of Newcastle, NSW 2308, Australia

e-mail: famb1es@yahoo.es

M. RIUS-FONT, Departament de Matemàtica Aplicada IV, Universitat Politècnica de Catalunya, C/Esteve Terrades 5, 08860 Castelldefels, Spain

e-mail: mrius@ma4.upc.edu 